\title{
Factors influencing food preparation behaviours: findings from focus groups with Mexican-American mothers in southern California
}

\author{
Teresa M Smith ${ }^{1,2, *}$, Genevieve F Dunton ${ }^{3}$, Courtney A Pinard ${ }^{1,2}$ and Amy L Yaroch ${ }^{1,2}$ \\ 'Gretchen Swanson Center for Nutrition, 8401 W. Dodge Road, Suite 100, Omaha, NE 681 14, USA: \\ ${ }^{2}$ Department of Health Promotion, Social \& Behavioral Health, University of Nebraska Medical Center, NE, USA: \\ ${ }^{3}$ Departments of Preventive Medicine and Psychology, University of Southern California, CA, USA
}

Submitted 14 July 2014: Final revision received 21 April 2015: Accepted 14 May 2015: First published online 14 August 2015

\begin{abstract}
Objective: The purpose of the present study was to explore food preparation behaviours, attitudes, meal planning and shopping among Mexican-American mothers. Design: Data were collected through four focus groups with mothers of Mexican origin/ancestry who considered themselves to be the primary food preparer. Topics included food preparation behaviours and influencers (culture, family, attitudes, barriers, meal planning and shopping). Data were analysed using a qualitative grounded theory approach. All focus groups were audio recorded, transcribed verbatim and coded for themes.

Setting: Data were collected in southern California, USA in 2013.

Subjects: Of the sample of twenty-one Mexican-American mothers, thirteen were born outside the USA and the mean household size was five members.

Results: Participants reported that food was often prepared using traditional staples and food preparation behaviours were learned from maternal family members. Participants also suggested that health was influenced by foods eaten and how they were prepared. Salient factors influencing food preparation behaviours included culture and tradition, maternal family members' food preparation behaviours, food preparation self-efficacy and attitudes towards healthy eating. Time and busy schedules were cited as barriers.

Conclusions: Future interventions should consider utilizing family-based approaches and teaching culturally relevant food preparation skills, especially to youth, while reinforcing more healthful dietary practices.
\end{abstract}

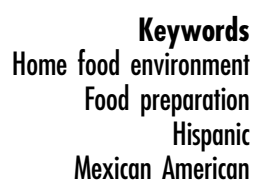

Obesity among Mexican Americans is a significant public health concern, as a recent study estimated that $37 \%$ of men and $42 \%$ of women are obese ${ }^{(1)}$, compared with $35 \%$ of all adults in the USA ${ }^{(2)}$. This high prevalence of obesity contributes to a disproportionate and increased risk for chronic diseases such as cancer ${ }^{(3)}$, hypertension and diabetes mellitus ${ }^{(1)}$ among Mexican Americans. Because Hispanics are the fastest-growing minority population in the USA and the largest sub-population are those of Mexican origin $(65 \%)^{(4)}$, obesity prevention interventions should consider culturally relevant approaches for Mexican Americans.

One main determinant of obesity across populations is poor dietary behaviour, such as low fruit and vegetable intake, added sugar and/or high fat intake. Specifically with regard to Mexican Americans, a 2009 study suggested that fruit and vegetable intake was higher among this group compared with other Hispanic subgroups and non-Hispanic white women, although the Mexican-American subgroup still failed to meet the recommended intake of 3.5-6.5 cups $/ \mathrm{d}^{(5,6)}$. A profile of 25 years of data (1982-2006) from variations of the National Health and Nutrition Examination Survey (NHANES) showed that total fat intake in Mexican Americans has decreased; most recently $32 \%$ of energy came from dietary fat, which falls within the recommended range of 20 to $35 \%$ of total energy ${ }^{(7,8)}$. However, total energy intake and carbohydrate intake have increased; with average energy intake increasing from 8297 to $9146 \mathrm{~kJ} / \mathrm{d}$ (1983 to $2186 \mathrm{kcal} / \mathrm{d}$ ) and percentage intake of carbohydrates rising from 46 to $51 \%{ }^{(8)}$.

Exploring ways to promote healthy food preparation in the home may support healthy eating and body weight among Hispanic Americans because the proportion of energy consumption from food prepared outside the 
home has increased in recent decades (from 18\% in $1977-78$ to $32 \%$ in 2006) ${ }^{(9)}$ and a recent study found that about $11 \%$ of total energy comes from fast-food intake among adults in the USA ${ }^{(10)}$. Previous studies suggest that cultural and familial approaches to healthy eating in the home food environment can contribute to increased fruit and vegetable intake and decreased fat intake among Hispanics ${ }^{(11-13)}$. Focusing on the home food environment, such as factors that may influence practices and parental policies around meal times and snacking ${ }^{(14,15)}$ and frequency of family meals ${ }^{(16,17)}$, is one strategy to consider in a multifaceted approach to increasing healthful eating among Mexican-American families.

More frequent and skilful food preparation has been shown to be associated with healthier dietary intake among young adults $^{(18)}$, adolescents ${ }^{(19)}$ and low-income women $^{(20)}$, although much of this work has not been conducted among racial or ethnic minority populations. Additionally, time spent in food preparation has been shown to be inversely related to BMI among racially and ethnically diverse samples of women ${ }^{(21,22)}$. Food preparation behaviours and related factors is an avenue within the home food environment that may improve overall dietary intake among families, yet is currently sparsely addressed in published literature ${ }^{(23)}$. A number of cooking-based programmes have shown that such interventions provide an opportunity for public health professionals to promote long-term dietary changes and subsequent health outcomes, although none of these was described as being developed or examined in a Hispanic population ${ }^{(24)}$. To the best of our knowledge, no published studies have qualitatively explored food preparation behaviours and related sociocultural factors among Mexican Americans.

The aim of the present study was to use a qualitative approach to investigate food preparation behaviours, attitudes, meal planning and shopping behaviours in a sample of Mexican-American mothers in southern California, which will provide a descriptive and subjective narrative from the participants themselves ${ }^{(25,26)}$. Our intent is that these qualitative results can help to inform the development of culturally appropriate dietary interventions among Hispanic Americans, and more specifically among Mexican Americans.

\section{Experimental methods}

For the present qualitative study, we conducted four focus group discussions with twenty-one Mexican-American mothers (size of focus groups ranged from two to eleven mothers) who considered themselves to be the primary food preparer in their household. The University of Nebraska Medical Center Institutional Review Board deemed that review was not required for the study. Participants received a $\$ \mathrm{US} 20$ gift card to a discount retail store as incentive for their participation.

\section{Participants and recruitment}

Hispanic parents/caregivers were recruited through purposive convenience sampling in Los Angeles and Riverside counties in southern California. This specific area was selected because of its large proportion of Hispanic individuals relative to the rest of the area's population $^{(27)}$. Each potential participant underwent a brief screener in order to determine eligibility according to the following criteria: age 18 years and older; Hispanic; parent or guardian of at least one child under the age of 18 years; and considered themselves to be the primary food preparer of their household. The US Office of Management and Budget states that Hispanic or Latino origin can be considered the 'heritage, nationality group, lineage, or country of birth of the person or the person's parents or ancestors before their arrival in the US ${ }^{,(28)}$. For the present study, Hispanic refers to those of Hispanic and Latino origin. Incidentally, the entire sample consisted of mothers who self-identified as Mexican through ancestry or origin. Before starting each session, participants were told that all information would be kept confidential, that participation was voluntary and that they could stop participation at any time without any consequences.

\section{Procedures}

Focus group research questions were developed to specifically examine: (i) family influence on food preference and preparation; (ii) meal planning and shopping; (iii) food preparation attitudes and self-efficacy for food preparation; and (iv) characteristics and behaviours of food preparation (see Appendix 1). Focus groups were conducted to the point of theoretical saturation, the point at which no new information or themes were observed in the data ${ }^{(29)}$. Two theories guided the development of the research questions: Family Systems Theory ${ }^{(30)}$ and Social Cognitive Theory ${ }^{(31)}$. The Family Systems Theory emphasizes that families live in complex systems in which multiple interactions occur simultaneously and has been identified as important in its presentation of the reciprocal influences within the home environment on health behaviours $^{(30,32)}$. Further, the Family Systems Theory elucidates the reciprocal relationship in which parental modelling influences child eating behaviours and child preferences influence meal planning and shopping choices in parents ${ }^{(33,34)}$. The Social Cognitive Theory addresses the reciprocal interplay between self-regulatory and environmental determinants of health behaviour ${ }^{(31)}$.

Each of the four focus groups was conducted by a trained moderator (three in English and one with a native Spanish speaker) using a standardized protocol of focus group questions and probes ${ }^{(35)}$. The three English focus groups were conducted in the commons area of a community dance studio and the Spanish focus group was conducted in the home of a community member. All focus groups were conducted during the weekends upon 
request of the participants. At the end of the focus group discussion, participants were asked to complete a short demographic questionnaire which asked about age, sex, country of origin/years in USA, household size, income, education, age of children, work status and marital status. Focus group sessions lasted approximately $30-45 \mathrm{~min}$ and refreshments were provided. All focus group sessions were audio recorded, transcribed verbatim and coded for themes. The focus group conducted in Spanish was transcribed in Spanish and translated into English by a trained Spanish speaker.

\section{Data analysis}

Two coders utilized the constant comparison methodology to create a coding scheme based on the focus group questions and responses ${ }^{(36)}$. They then reviewed the codes and determined that consensus had been reached and there were no discrepancies. Data analysis was conducted using the qualitative software package QSR NVivo version 10. Transcriptions were coded into conceptual categories and emergent themes were identified through frequency of coding within similar contexts across focus groups.

\section{Results}

Twenty-one Mexican-American mothers participated in the focus groups (see Table 1). The mean age of participants was 35 years (range: 25-46 years) and the mean household size was five members (range: $2-10$ members). Most participants were married $(n 13)$ and reported a wide range of ages of children in their homes. Most of the participants ( $n$ 18) held at least a high-school degree or GED (General Educational Development), about half ( $n$ 9) reported they primarily spoke English at home, about half ( $n$ 10) reported being employed full time and just under half ( $n 8)$ reported being born in the USA. There was a wide range of household incomes reported.

\section{Emergent themes}

Six salient themes emerged from the focus groups and included: (i) cultural and traditional influence; (ii) social and familial influence; (iii) food preparation self-efficacy; (iv) attitudes towards health and healthy eating; (v) meal planning and shopping; and (vi) barriers related to time and busy schedules. Themes and relevant quotes are listed in Appendix 2.

\section{Cultural and traditional influence}

Overall, participants emphasized the influence of their Mexican-American culture and upbringing on their dietary behaviours. When describing culturally important foods prepared at home, one participant said, 'You always have to have beans and tortillas.' Other traditional staple foods
Table 1 Characteristics of Mexican-American mothers in focus groups exploring food preparation behaviours and related factors (n 21), southern California, USA, 2013

\begin{tabular}{|c|c|c|}
\hline Demographic variable & Frequency & $\%$ \\
\hline \multicolumn{3}{|l|}{ Education } \\
\hline$<$ High-school degree & 1 & 4.8 \\
\hline High school degree or GED & 10 & 47.6 \\
\hline College degree & 8 & $38 \cdot 1$ \\
\hline Missing & 2 & 9.5 \\
\hline \multicolumn{3}{|l|}{ Household annual income (\$US) } \\
\hline$<34999$ & 4 & $19 \cdot 1$ \\
\hline $35000-74999$ & 4 & $19 \cdot 1$ \\
\hline$\geq 75000$ & 5 & $23 \cdot 8$ \\
\hline Missing & 8 & 38.1 \\
\hline \multicolumn{3}{|l|}{ Household language } \\
\hline English only & 9 & 42.9 \\
\hline Spanish only & 4 & $19 \cdot 1$ \\
\hline English and Spanish & 7 & $33 \cdot 3$ \\
\hline Missing & 1 & 4.8 \\
\hline \multicolumn{3}{|l|}{ Employment status } \\
\hline Employed full time & 10 & 47.6 \\
\hline Employed part time & 2 & 9.5 \\
\hline Homemaker & 7 & 33.3 \\
\hline Missing & 2 & 9.5 \\
\hline \multicolumn{3}{|l|}{ Marital status } \\
\hline Married & 13 & 61.9 \\
\hline Divorced or never married & 6 & 28.6 \\
\hline Missing & 2 & 9.5 \\
\hline \multicolumn{3}{|l|}{ Born in the USA } \\
\hline Yes & 8 & $38 \cdot 1$ \\
\hline No & 10 & $47 \cdot 6$ \\
\hline Missing & 3 & 14.3 \\
\hline \multicolumn{3}{|c|}{ One or more child in household aged $0-5$ years } \\
\hline Yes & 14 & 66.7 \\
\hline No & 6 & 28.6 \\
\hline Missing & 1 & 4.8 \\
\hline \multicolumn{3}{|c|}{ One or more child in household aged $6-12$ years } \\
\hline Yes & 16 & $76 \cdot 2$ \\
\hline No & 4 & $19 \cdot 0$ \\
\hline Missing & 1 & 4.8 \\
\hline \multicolumn{3}{|c|}{ One or more child in household aged $13-17$ years } \\
\hline Yes & 7 & 33.3 \\
\hline No & 11 & 52.4 \\
\hline Missing & 3 & $14 \cdot 3$ \\
\hline
\end{tabular}

GED, General Educational Development.

and ingredients included: vegetables (e.g. corn, tomato, onion, broccoli, lettuce, carrots, celery, cucumber and potato), fruits (e.g. apple, banana, mango, watermelon and papaya), chicken, fish, white rice, oil, lard, salt, spices and chillies. Sopa (soup) was described as a culturally significant dish. To prepare it, one participant said, 'You start off with a little diced onion and tomato in the pan and you get the Mexican brand bouillon.' Other dishes commonly prepared included tacos, enchiladas, tostados, burritos, and fruit water or fruit juice. When preparing foods and dishes with fat, participants described generally using oil more commonly than lard or butter. Participants reported also regularly preparing American-style dishes (e.g. macaroni and cheese or casseroles), describing them as favoured by their families with opportunities to incorporate healthy ingredients (e.g. vegetables and whole-wheat pasta). 


\section{Social and familial influence}

Mexican-American mothers in the current study reported maternal family members influencing their food preparation behaviours, citing that their mothers, aunts and grandmothers prepared full meals daily during their childhoods. One participant said, 'Mom always pulled me into the kitchen, so I'm comfortable in the kitchen.' These maternal influences appeared to track into the present day, as participants reported that they continued to learn about food preparation from maternal family members (including mothers-in-law). Participants did not overtly describe teaching their children how to prepare food. They described observation as a key element to their own learning process, as maternal family members tended not to use measuring utensils or follow recipes, making most dishes and meals 'to taste'. While greatly influenced by the behaviours and attitudes of their maternal family members, participants did not necessarily perceive themselves needing to prepare food in the same way, noting that they often attempted to prepare food and meals in healthier ways (e.g. less fat or salt) than they were taught. One participant said:

'I learned [to cook] from [my mom] and you know, it's not the way I cook, but her food is delicious, sometimes when I watch her cook she uses a lot of salt and my cooking probably is a lot more bland.'

The participants also cited that their families were large and involved frequent social events, such as a fiesta, Quinceañera or wedding, which feature spreads of homemade dishes.

\section{Food preparation self-efficacy}

A prominent theme that emerged from the focus group data was that self-efficacy was related to food preparation. As described in the previous section, the food preparation behaviours of Mexican-American mothers were largely influenced by the behaviours and attitudes of their maternal family members. Participants described utilizing the skills and behaviours of maternal family members as a barometer for setting outcome expectations for their own food preparation skills and behaviours. One participant said:

'It's really hard to sort of match my mother-in-law, I always know [food I prepare] is not going to taste like hers, and once I got over that, I'm more willing to make mistakes, and now I'm getting more confident, but I had to sort of fail.'

Because participants largely saw their own skills as lacking in comparison to their maternal family members, they described themselves as inadequate cooks or simply being unable to cook. Another participant said:

'I can't cook, especially coming off when your mom cooks really well and your mother-in-law cooks really well, you're like I'm not even going to try,
I had no confidence ... I sort of had to break free and gain confidence ... it is hard when you have that cooking dynasty of your parents.'

\section{Attitudes towards bealth and bealthy eating}

Under the theme of attitudes towards health and healthy eating, two sub-themes emerged: foods eaten influence health and perceived burden associated with food preparation.

\section{Foods eaten influence bealth}

Participants expressed shared beliefs that foods eaten influence health, both in the short term (e.g. providing energy necessary for daily living) and long term (e.g. contributing to the prevention or development of chronic disease), although opinions on long-term health outcomes were especially prominent. Many participants were expressly concerned about their own risk, or a family member's risk, for diabetes. One participant said, 'My husband is prone to being diabetic and other diseases, so I try to cook healthier because of him.' Participants indicated that they believed foods prepared at home were generally healthier than any foods or meals consumed from other venues. Another participant said, 'I think if you make it, I still think it's better than if you go out.'

\section{Perceived burden associated with food preparation}

In some cases, participants felt burdened by trying to strike a balance between maintaining cultural values and ensuring their family members ate healthily. They described feeling guilt associated with occasions of incorporating, but also occasions of not incorporating, certain less healthy ingredients into meals (e.g. lard and sugar) that were desired by their family members. One participant described:

'When I buy lard, I feel bad, but one little box will last me like a good 8 months.'

This burden could perhaps stem from their perception that traditional Mexican foods and meals are high in fat and salt. One participant said:

'I don't eat Mexican food outside of my mom's kitchen, she doesn't care about fat content, she's an overweight, typical Mexican woman ... I think because we grew up that way, I'm going to feed my kids right.'

Many participants described attempting to cook healthier for their families compared with foods that were prepared for them during childhood, causing some tension among generations. Another participant described:

'I get a lot of backlash, too, because I try to eat super healthy and being like, Mexican, they eat really bad, my mom still cooks with lard ... we're not fine, we're all diseased.' 


\section{Meal planning and shopping}

Participants consistently reported they were responsible for shopping and meal planning for their families. In general, participants described grocery shopping weekly and purchasing food from more than one store. One participant described what influenced the foods that she purchased, 'Whether they are going to eat it, whether or not I am going to cook it, and how long it's going to last in my fridge.' Cost of food was described as minimally influential among this sample, although strategies for purchasing staple items in bulk were discussed. Also, participants described that they perceived it being easy to purchase healthy foods in their neighbourhoods.

\section{Time and busy schedules as barriers to bome food preparation}

Limited time and busy schedules for each of the family members (e.g. parent work schedules and children's activities) were consistently described as barriers to food preparation in the home. As a result, participants described preparing meals that they perceived to be quick and easy, with which their families were familiar. Although not opposed to trying new recipes or dishes, they cited that it was challenging to find the time necessary and there was risk that presenting an unfamiliar dish to their family would result in them not liking and/or eating it. One participant said, 'It takes time and it's hard to find the time, so you go to what you already know how to make.' Accordingly, eating food from a restaurant was described as an easier option for their families. Parents also described regularly utilizing prepared meals from grocery stores as a relatively quick and healthy alternative to preparing food at home. Participants indicated that it usually takes them 20 to $60 \mathrm{~min}$ when preparing a dish or meal, and they explained they had more time available to prepare food during the weekends compared with weekdays. When asked to elucidate on what 'cooking from scratch' meant to them, they described buying whole ingredients (e.g. grains, produce and raw meat) and combining and cooking them. Pre-packaged foods, such as bagged lettuce, were not often utilized. Despite barriers, participants said they tried to eat as many meals at home as possible, as they valued the healthfulness of foods and meals prepared at home, as well as the time spent eating with their family.

\section{Discussion}

Given the importance that the home food environment plays in obesity and disease prevention, it is critical to understand what aspects of food preparation behaviours are attributed to cultural, social and familial environments ${ }^{(13)}$. MexicanAmerican mothers in the current study reported several salient factors influencing their food preparation behaviours, most notably culture and tradition, family, food preparation self-efficacy and attitudes towards healthy eating.
In the USA, the family unit is typically considered the small, household-based family of parents and children ${ }^{(37)}$. However, Mexican-American mothers in the current study explained that as the primary food preparer of their household, they prepared food for their children, spouse/ partner, parents, in-laws and siblings; each having a significant role in their family unit. This structure may be a result of cultural familistic values, socio-economic circumstances or evidence of continuously shifting family dynamics ${ }^{(38,39)}$. Accordingly, participants felt responsible for regulating the foods procured, prepared, served and eaten by family members and expressly described often trying to prepare foods in more healthful ways to reduce risk for chronic diseases, in particular diabetes, among family members. Future interventions that aim to increase healthy eating through food preparation among Mexican Americans should consider incorporating family-based approaches within the home.

Participants set outcome expectations of food preparation for themselves based on how they viewed the food preparation skills and behaviours of maternal family members. When they did not meet expectations, their selfefficacy for food preparation appeared to be adversely affected. Increasing self-efficacy through activities such as through mastery or vicarious experiences ${ }^{(40)}$ among Mexican-American mothers may influence the frequency and healthfulness of meals prepared at home. Woodruff and Kirby indicated that self-efficacy is a key factor in frequency of cooking ${ }^{(41)}$ and the current study suggests self-efficacy of food preparation may be an important influencer of how healthily foods are prepared ${ }^{(31)}$. Food preparation and cooking interventions specifically targeting younger Mexican-American generations may aid in the mastery of food preparation in an evolving food environment, while maintaining important cultural traditions.

Other key studies have qualitatively explored Hispanic mothers' diet-related behaviours in the home food environment $^{(42,43)}$. Much like the current study, these studies demonstrated that maternal family members play a significant role in the food preparation for a family ${ }^{(42,43)}$. The current study describes how this sample of MexicanAmerican mothers attempted to prepare food and meals in more healthful ways than their maternal family members. This is potentially burdensome, as they conveyed attempting to maintain culture while also feeding their families more healthful foods, for which they sometimes received negative feedback from family members. A study that qualitatively assessed food preparation behaviours of a sample of Latina women also found they tended to use traditional staple items to prepare dishes and meals that were familiar to them and their families ${ }^{(42)}$, which could potentially provide an opportunity for incorporating healthier foods (e.g. fruits or vegetables) and/or reducing less healthful foods (e.g. ones with added sugar or salt). Participants of the current study stated that they prepared dishes that were traditional to their Mexican culture, but 
also frequently prepared American-style dishes, describing them as fast and easy with opportunities to incorporate healthy ingredients. This idea is somewhat contradictory to studies that have asserted that less traditional dietary patterns may be associated with poorer diet ${ }^{(44,45)}$ and suggests both traditional and American-style dishes may lend themselves to occasions to incorporate more healthful food preparation behaviours. Tailoring homebased dietary interventions to utilize traditional foods, along with American-style foods, may garner more attention and uptake among a Mexican-American population.

Participants indicated that they believed foods prepared at home are generally healthier than any foods or meals consumed from other venues, which is confirmed by several studies that have found a relationship between healthy eating outcomes and eating at home ${ }^{(33,46-48)}$. However, time and busy schedules were cited as barriers to preparing food at home among this sample. Regrettably, in the last 50 years US adults have both decreased intake of foods prepared at home and reduced time spent cooking ${ }^{(8)}$, and energy consumed from food prepared outside the home has increased from $18 \%$ in $1977-78$ to $32 \%$ in $2006^{(9)}$. Strategies for swapping more healthful food preparation behaviours for less healthy ones in common and familiar Mexican and American-style dishes may improve diet without involving additional time, especially among more acculturated families. Given previous studies that have demonstrated relationships among acculturation (which can be affected by ethnicity, country of origin, religion, ideals, characteristics or behaviours) and decline of more healthful dietary intake among Mexican Americans ${ }^{(45,49)}$, future research should consider how these barriers, as well as attitudes and beliefs, differ among acculturation and income levels.

The present qualitative research study has some limitations to be considered. First, the focus groups were designed to be interactive group discussions, lending to a less controlled environment, although they did allow for a discussion about cultural norms and social influence to occur in a naturalistic social setting (the 'group effect') ${ }^{(35,50)}$. Second, because data were largely interpreted by the authors of the study, caution was exercised by striving for objectivity and employing two independent coders to diminish potential interpretation bias. This practice reduces the potential for authors to interpret findings in the context of their personal attitudes, beliefs and experiences. Third, the study utilized a sample with an income and education distribution that suggests it was comprised of individuals of a relatively higher socio-economic status when compared with Mexican Americans nationally ${ }^{(51)}$, potentially limiting generalizability of some of the factors identified in the study to higher-income populations. Further, acculturation was not measured in this group and nor were participants selected on this basis, so there was not an ability to distinguish factors between levels of acculturation. Given that acculturation has been shown to be related to the healthfulness of dietary intake among Mexican Americans, it may also have an influence on the healthfulness of food preparation behaviours and should be objectively measured in future research. Despite these limitations and in light of the sample size, the homogeneity among the group lent to more consistent findings. Further, the standardized protocol of questions and probes contributed to in-depth exploration of topics initiated by participants within focus group sessions. Lastly, the study contributes to dearth in the literature by describing food preparation behaviours and related factors among Mexican-American mothers in southern California.

\section{Implications for research and practice}

The present study sought to better understand various factors related to dietary behaviours among MexicanAmerican families. Preliminary information was obtained to help characterize the home food environment specific to Mexican-American families, underscoring how food preparation in the home can support a healthy diet. Future interventions should consider adopting a family-based approach and incorporate culturally relevant, as well as common and familiar, food preparation skills while reinforcing more healthful dietary practices. Further, future research should seek to develop and incorporate food preparation and associated factors into dietary surveillance and assessment.

\section{Acknowledgements}

Acknowledgements: The authors thank Clyde 'Cappy' Pentz, Lecturer in the Department of Psychology at California State University, Long Beach, for his enthusiastic support and help with recruiting undergraduate research assistants for this study. The authors also thank Anay Salgado and Maria Leon, students in the department of Psychology at California State University, Long Beach, for their spirited help with recruitment, translation and leading focus groups. The authors also thank all parents who participated in the focus groups. Financial support: The project described was supported by the National Cancer Institute (grant number R25CA112383). The content is solely the responsibility of the authors and does not necessarily represent the official views of the National Cancer Institute of the National Institutes of Health. The National Institutes of Health had no role in the design, analysis or writing of this article. Conflict of interest: There are no known conflicts of interest to this study. Authorship: All authors contributed to the conception of this work. T.M.S. designed, conducted the data collection, analysed the data and wrote the manuscript; T.M.S. and G.F.D. designed the focus group questions and probes and oversaw recruitment efforts. G.F.D., C.A.P. and A.L.Y. provided extensive guidance throughout the study and writing of the manuscript. Ethics of buman subject 
participation: The University of Nebraska Medical Center Institutional Review Board deemed that review was not required for this study.

\section{References}

1. Daviglus ML, Talavera GA, Avilés-Santa M et al. (2012) Prevalence of major cardiovascular risk factors and cardiovascular diseases among Hispanic/Latino individuals of diverse backgrounds in the united states. JAMA 308, 1775-1784.

2. Ogden CL, Carroll MD, Kit BK et al. (2014) Pevalence of childhood and adult obesity in the United States, 2011-2012. JAMA 311, 806-814.

3. Vucenik I \& Stains JP (2012) Obesity and cancer risk: evidence, mechanisms, and recommendations. Ann $N Y$ Acad Sci 1271, 37-43.

4. US Census Bureau (2012) 2011 American Community Survey: Table B03001. http://factfinder.census.gov/faces/ tableservices/jsf/pages/productview.xhtml?src=bkmk (accessed March 2014).

5. Dietary Guidelines Advisory Committee (2010) Report of the Dietary Guidelines Advisory Committee on the Dietary Guideline for Americans, 2010, to the Secretary of Agriculture and the Secretary of Health and Human Services. Washington, DC: US Department of Agriculture, Agricultural Research Service; available at http://www.cnpp.usda.gov/ sites/default/files/dietary_guidelines_for_americans/2010D GACReport-camera-ready-Jan11-11.pdf

6. Colón-Ramos U, Thompson FE, Yaroch AL et al. (2009) Differences in fruit and vegetable intake among Hispanic subgroups in California - results from the 2005 California Health Interview Survey. J Am Diet Assoc 109, 1878-1885.

7. Division of Nutrition, Physical Activity, and Obesity \& Centers for Disease Control and Prevention (2012) Nutrition for Everyone: Basics: Dietary Fat. http://www.cdc.gov/ nutrition/everyone/basics/fat/ (accessed June 2014).

8. Smith LP, Ng SW \& Popkin BM (2013) Trends in US home food preparation and consumption: analysis of national nutrition surveys and time use studies from 1965-1966 to 2007-2008. Nutr J 12, 45.

9. Todd J \& Mentzer Morrison R (2014) Less Eating Out, Improved Diets, and More Family Meals in the Wake of the Great Recession. Washington, DC: US Department of Agriculture, Economic Research Service.

10. Fryar CD \& Ervin RB (2013) Caloric Intake from Fast Food among Adults: United States, 2007-2010. NCHS Data Brief no. 114. Hyattsville, MD: National Center for Health Statistics.

11. Flores G, Maldonado J \& Durán P (2012) Making tortillas without lard: Latino parents' perspectives on healthy eating, physical activity, and weight-management strategies for overweight Latino children. I Acad Nutr Diet 112, 81-89.

12. Committee on Accelerating Progress in Obesity Prevention \& Institute of Medicine (2012) Measuring Progress in Obesity Prevention: Workshop Report. Washington, DC: The National Academies Press.

13. Sanjur D (1995) Hispanic Foodways, Nutrition, and Health. Boston, MA: Allyn and Bacon.

14. Wyse R, Campbell E, Nathan N et al. (2011) Associations between characteristics of the home food environment and fruit and vegetable intake in preschool children: a crosssectional study. BMC Public Health 11, 938.

15. Rodríguez-Oliveros G, Haines J, Ortega-Altamirano D et al. (2011) Obesity determinants in Mexican preschool children: parental perceptions and practices related to feeding and physical activity. Arch Med Res 42, 532-539.

16. Van der Horst K, Oenema A, Ferreira I et al. (2007) A systematic review of environmental correlates of obesityrelated dietary behaviors in youth. Health Educ Res 22, 203-226.
17. Sobal J \& Hanson K (2014) Family dinner frequency, settings and sources, and body weight in US adults. Appetite 78, 81-88.

18. Larson NI, Perry CL, Story M et al. (2006) Food preparation by young adults is associated with better diet quality. $J \mathrm{Am}$ Diet Assoc 106, 2001-2007.

19. Larson NI, Story M, Eisenberg ME et al. (2006) Food preparation and purchasing roles among adolescents: associations with sociodemographic characteristics and diet quality. J Am Diet Assoc 106, 211-218.

20. McLaughlin C, Tarasuk V \& Kreiger N (2003) An examination of at-home food preparation activity among low-income, food-insecure women. J Am Diet Assoc 103, 1506-1512.

21. Zick CD, Stevens RB \& Bryant WK (2011) Time use choices and healthy body weight: a multivariate analysis of data from the American Time use Survey. Int J Behav Nutr Phys Act 8, 84 .

22. Kolodinsky JM \& Goldstein AB (2011) Time use and food pattern influences on obesity. Obesity (Silver Spring) 19, 2327-2335.

23. Mello JA, Gans KM, Risica PM et al. (2010) How is food insecurity associated with dietary behaviors? An analysis with low-income, ethnically diverse participants in a nutrition intervention study. J Am Diet Assoc 110, 1906-1911.

24. Reicks M, Trofholz AC, Stang JS et al. (2014) Impact of cooking and home food preparation interventions among adults: outcomes and implications for future programs. J Nutr Educ Behav 46, 259-276.

25. Tashakkori A \& Teddlie C (2003) Handbook of Mixed Methods in Social \& Behavioral Research. Thousand Oaks, CA: SAGE Publications Inc.

26. Creswell JW (2013) Research Design: Qualitative, Quantitative, and Mixed Methods Approaches. Thousand Oaks, CA: SAGE Publications Inc.

27. Ennis SR, Rios-Vargas M \& Albert NG (2011) The Hispanic Population: 2010. 2010 Census Briefs. http://www.census.gov/ prod/cen2010/briefs/c2010br-04.pdf (accessed March 2013).

28. Office of Management and Budget (1997) Revisions to the Standards for the Classification of Federal Data on Race and Ethnicity. Federal Register Notice. https://www.whitehouse. gov/omb/fedreg_1997standards (accessed December 2013).

29. Guest G, Bunce A \& Johnson L (2006) How many interviews are enough? An experiment with data saturation and variability. Field Methods 18, 59-82.

30. Broderick CB (1993) Understanding Family Process: Basics of Family Systems Theory. Thousand Oaks, CA: SAGE Publications Inc.

31. Bandura A (1998) Health promotion from the perspective of social cognitive theory. Psychol Health 13, 623-649.

32. White JM \& Klein DM (2008) Family Theories. Thousand Oaks, CA: SAGE Publications Inc.

33. Berge JM, MacLehose RF, Loth KA et al. (2012) Family meals. Associations with weight and eating behaviors among mothers and fathers. Appetite 58, 1128-1135.

34. Ayala GX, Carnethon M, Arredondo E et al. (2014) Theoretical foundations of the Study of Latino (SOL) Youth: implications for obesity and cardiometabolic risk. Ann Epidemiol 24, 36-43.

35. Hennink M, Hutter I \& Bailey A (2010) Qualitative Research Methods. London: SAGE Publications Ltd.

36. Lewis-Beck MS, Bryman AE \& Liao TF (2003) The Sage Encyclopedia of Social Science Research Methods. Thousand Oaks, CA: SAGE Publications Inc.

37. Cherlin AJ (2009) Public and Private Families: An Introduction. New York: McGraw-Hill.

38. Landale NS, Oropesa RS \& Bradatan C (2006) Hispanic families in the United States: family structure and process in an era of family change. In Hispanics and the Future of America, pp. 138-178 [M Tienda and F Mitchell, editors]. Washington, DC: The National Academies Press. 
39. Dvoráková-Janů V (2000) Changing circumstances for accepting of behavioral patterns in connection with food preparation. Acta Univ Carol Med (Praba) 41, 127-130.

40. Bandura A (1994) Self-efficacy. In Encyclopedia of Human Behavior, vol. 4, pp. 71-81 [VS Ramachaudran, editor]. New York: Academic Press.

41. Woodruff SJ \& Kirby AR (2013) The associations among family meal frequency, food preparation frequency, selfefficacy for cooking, and food preparation techniques in children and adolescents. J Nutr Educ Behav 45, 296-303.

42. Ayala GX, Elder JP, Campbell NR et al. (2001) Nutrition communication for a Latino community: formative research foundations. Fam Community Health 24, 72-87.

43. Martinez SM, Rhee K, Blanco E et al. (2015) Maternal attitudes and behaviors regarding feeding practices in elementary school-aged Latino children: a pilot qualitative study on the impact of the cultural role of mothers in the US-Mexican border region of San Diego, California. J Acad Nutr Diet 115, Suppl. 5, S34-S41.

44. Flores M, Macias N, Rivera M et al. (2010) Dietary patterns in Mexican adults are associated with risk of being overweight or obese. J Nutr 140, 1869-1873.

45. Colby SE (2013) Multicultural food perspectives: strategies for health care providers. Am J Lifestyle Med 7, 13-19.
46. Ma Y, Bertone ER, Stanek EJ et al. (2003) Association between eating patterns and obesity in a free-living US adult population. Am J Epidemiol 158, 85-92.

47. Utter J, Denny S, Robinson E et al. (2013) Family meals among New Zealand young people: relationships with eating behaviors and body mass index. J Nutr Educ Behav 45, 3-11.

48. Gross SM, Pollock ED \& Braun B (2010) Family influence: key to fruit and vegetable consumption among fourth- and fifth-grade students. J Nutr Educ Behav $\mathbf{4 2}$, 235-241.

49. Neuhouser ML, Thompson B, Coronado GD et al. (2004) Higher fat intake and lower fruit and vegetables intakes are associated with greater acculturation among Mexicans living in Washington State. J Am Diet Assoc 104, $51-57$.

50. Morgan DL (1996) Focus groups. Annu Rev Sociol 22, 129-152.

51. Gonzalez-Barrera A \& Lopez MH (2013) A demographic portrait of Mexican-origin Hispanics in the United States. Pew Research Center Hispanic Trends. http://www. pewhispanic.org/2013/05/01/a-demographic-portrait-ofmexican-origin-hispanics-in-the-united-states/ (accessed July 2014). 


\section{Appendix 1}

Structural model of food preparation behaviours and related factors and corresponding focus group questions administered to Mexican-American mothers living in southern California, USA, 2013

Structural factor
Family influence on food preference
and preparation
Meal planning and shopping
Food preparation attitudes and self-
efficacy

Focus group questions

- Who in your household does most of the food preparation and cooking? How much time do you spend cooking/preparing meals for your family? How much do other family members or your children help cook? What meals do you eat together as a family? Please describe sample breakfast (lunch, dinner) meals that you prepare for your family.

- What foods are important to you, your family, friends and neighbours? How important to you is it that you get to eat these foods?

- In what ways are your food choices and eating patterns shaped by your social activities, and how do you respond to these pressures and opportunities? Relationships with family and friends?

Community events, such as neighbourhood fairs, parties, church functions?

- Do you try to role model any eating-related behaviours for your family?

- Does your family typically eat meals together? (If no, why not?)

- How do you decide what meals/snacks to prepare for your family?

- In what ways is it easy or hard to get your family to eat healthy foods? How do the adults' preferences or habits influence eating healthy foods? How do the children's preferences or habits influence eating healthy foods?

- At meals, how does your family determine portion size?

- Do you ever serve leftovers as meals/snacks?

- Do you have adequate resources in your home for preparing meals?

- Who typically does the grocery shopping in your household? Where do you (or person responsible) shop?

- What are some of the factors that determine what types of groceries are bought for your family?

- In what ways is it easy or hard to get healthy foods for your family or household?

- What are some of your favourite meals to make for your family?

- Have you ever served your family a new dish or meal?

- Do you ever prepare different foods for different family members for one meal?

- Do you think what you feed your family affects their health? Are there any foods you make an extra effort to feed them for health reasons? Are there any foods that you try to avoid preparing?

- What are some of the reasons why you might eat fast food or from restaurants over preparing food at home?

- Do you think that making meals at home helps you eat more healthily?

- What rules does your family have at meal time?

- How confident are you in your ability to prepare meals at home for your family? How about healthy meals? How about meals on a budget?

- How did you learn to prepare meals at home? In what ways are you passing your knowledge along to your kids?

Characteristics and behaviours of

- How often do you make homemade meals 'from scratch'? What does this mean to you?

food preparation

- When preparing meals at home what types of meats, poultry, or fish do you usually use? What fruits do you usually use/serve? What vegetables do you usually use/serve? What types of fats do you usually use/serve? What grains do you usually use/serve? What beverages do you usually serve?

- Describe how your culture influences what meals or recipes you make.

- How often do you use ready-made products?

- How often do you prepare meals using convenience items?

- How often does your family eat homemade meals at others' homes? 
Appendix 2

Themes and selected relevant quotes related to food preparation behaviours and related factors of MexicanAmerican mothers (n 21) living in southern California, USA, 2013

\begin{tabular}{ll}
\hline Theme & Selected quotes \\
\hline Cultural and traditional influence & 'You always have to have beans and tortillas.' \\
'l'll wipe some [lard] on my Comal with a napkin to trick them that like look you still got a \\
little slab and it still gives it the taste.' \\
'[Describing preparing sopa/soup] You start off with a little diced onion and tomato in the \\
pan and you get the Mexican brand bouillon.' \\
'I make easy stuff, too, like casseroles.' \\
'Boneless chicken, salmon, occasionally macaroni and cheese.'
\end{tabular}

Social and familial influencers

Food preparation self-efficacy

Attitudes towards health and healthy eating

Meal planning and shopping

Time and busy schedules as barriers
'Mom always pulled me into the kitchen, so, I'm comfortable in the kitchen.'

'I learned it from [my mom] and you know, it's not the way I cook ... she uses a lot of salt and my cooking probably is a lot more bland.'

'I try to cooking only with turkey, chicken, and fish ... When I go to a fiesta, or a Quinceañera, or a wedding, I'm going to have red meat, so I am going to have red meat regardless, so that's why l'm like l'm just going to stick to the turkey, the chicken and the fish.'

'It's really hard to sort of match my mother-in-law, I always know it's not going to taste like her, and once I got over that, I'm more willing to make mistakes, and now I'm getting more confident, but I had to sort of, fail, and ... I can tweak it and then I feel confident, so I think l'm more confident now.'

'I can't cook, especially coming off when your mom cooks really well and your mother-inlaw cooks really well, you're like I'm not even going to try, I had no confidence ... I sort of had to break free and gain confidence ... it is hard when you have that cooking dynasty of your parents and everything.'

Foods eaten influence health

'My husband is prone to being diabetic and other diseases, so I try to cook healthier because of him.'

'I think if you make it, I still think it's better than if you go out.'

Perceived burden associated with food preparation

'I'm guilty of still having lard in my refrigerator, I still do ... it's just that I'm used to but when I buy lard, I feel bad, but one little box will last me like a good 8 months.'

'I get a lot of backlash, too, because I try to eat super healthy and being like, Mexican, they eat really bad, my mom still cooks with lard, no we're not fine, we're all diseased, we didn't grow up well, I get a backlash for that.'

'I don't eat Mexican food outside of my mom's kitchen, she doesn't care about fat content, she's an overweight, typical Mexican woman, and she went to the doctor and he told her [that] her life expectancy is reduced because she is overweight ... I think because we grew up that way, I'm going to feed my kids right.'

'It's easy, there's healthy foods everywhere you just have to choose to buy them instead of junk or, I think for me it's easy to buy if you know what's healthy.'

'Whether they are going to eat it, whether or not I am going to cook it, and how long it's going to last in my fridge [determine what types of groceries bought].'

'We try to eat dinner together but with our schedule a lot of times it's just the two kids with one parents b/c we have so many things going on but we do try to eat dinner together.'

'Weeknights are out of the question, homework has to be done ... it's a big family, Mexican families are big, there's always things to do.'

'Other than the time, you know how to prepare, it takes time and it's hard to find the time, so you go to what you already know how to make.'

'You go to what's fast and what's there.'

'You just put everything together [to make lasagne] and its sort of healthy because I use wheat pasta and I use ground turkey but I don't make my sauce out of scratch I don't have time for that and then put it in the oven.' 